\title{
TV Broadcast Efficiency in 5G Networks from Subscriber Prospective
}

\author{
Chun Pong Lau and Basem Shihada \\ Computer, Electrical and Mathematical Science Engineering Division \\ King Abdullah University of Science and Technology (KAUST) \\ \{lau.pong, basem.shihada\}@kaust.edu.sa
}

\begin{abstract}
The flexibility of radio access network facilitated by 5G networks opens the gateway of deploying dynamic strategies for broadcasting TV channels in an efficient way. Currently, spectrum efficiency and bandwidth efficiency are the two common metrics measuring the efficiency of a system. These metrics assess the net bitrate for a unit of spectrum bandwidth. However, there is a lack of measurement, quantifying the effectiveness of a broadcasting strategy from the user perspective. In this paper, we introduce a novel measurement approach, called broadcast efficiency which considers the mobile user as a main reference. Broadcast efficiency is calculated as the number of served audiences per unit of radio resource. From numerical analysis, we show that broadcasting unpopular TV channels dramatically decreases the broadcast efficiency. This finding is evaluated by employing multiple distributions on the size of audience among TV channels. Furthermore, by conducting a real-life simulation, we discover that a high broadcast efficiency may result in a low percentage of served audiences if the audiences of TV channels are quite evenly distributed.
\end{abstract}

Index Terms-TV, broadcast, efficiency, 5G.

\section{INTRODUCTION}

After the successful operation of the fourth-generation (4G) networks, researchers are now enthusiastically designing the next generation mobile networks with even greater capability. With the support of Evolved MBMS (eMBMS) in Long-Term Evolution (LTE) networks, service providers are able to offer broadcast and multicast services to mobile subscribers [1]. Currently, mobile service providers are launching multimedia services to their subscribers. For example, in Saudi Arabia, the major operator Mobily has launched a new broadcasting service known as ' $m$ View' to deliver live television (TV) and video on demand services since 2014 [2]. In US, Verizon utilizes the 4G LTE networks to provide wireless TV [3]. In 2014, Nokia successfully launched a trial TV broadcasting service on LTE network in Munich, Germany [4]. When shaping the concept of the future $5 \mathrm{G}$ networks, researchers expect at least more than a thousand times of system capacity and ten times more spectral efficiency than the $4 \mathrm{G}$ networks [5]. By utilizing these capabilities, broadcasting TV channels on $5 \mathrm{G}$ networks becomes attractive and profitable in the short coming future.

In the current mobile network TV broadcasting service, service providers offer tens of TV channels for audiences to select from. The bitrate of each TV channel requires several hundreds to thousands kilobits per second. It is a challenge for mobile operators to simultaneously broadcast all of the TV channels to audiences within the limited amount of available bandwidth in each cell. Reported in [6], in order to broadcast $60 \mathrm{TV}$ channels in suburban area, a spectrum of more than $400 \mathrm{MHz}$ is required to deliver quality service in the worst scenario. It is almost impossible, as normally mobile service providers are not licensed with sufficient bandwidth to fulfill this requirement. Besides, researchers proposed cross-layer systems to reduce bandwidth consumption, such as superposition coded multicasting (SCM). In SCM, the base station (BS) superimposes two video streams, both good and fair quality streams of a video, in one radio signal to broadcast [7], [8]. Although this reduces the radio resource consumption for broadcasting multiple copies of the same video stream and ensure reliable receptions for users in various channel qualities, it does not reduce bandwidth for broadcasting multiple TV channels [9].

It is well known in literature that spectrum or bandwidth efficiency is the common approach used to gauge the rate that information is transmitted with a given unit of radio resources. However, in a broadcasting scenarios, this is no longer sufficient to quantify the effectiveness of a broadcasting strategy from the user perspective since the broadcasting strategies will influence the system performance when the size of audience is varying. In [10], Ye et al. introduced a metric to measure the effectiveness of broadcasting in vehicular adhoc network. However, their measurement is restricted to measure the number of nodes per unit time, which does not take the transmission bitrate into account. In [11], the broadcast efficiency is defined as the number of successfully received packets in a unit duration regardless of the senders. On the other hand, in [12], the broadcast efficiency is defined as the number of newly covered nodes reached per unit transmit power in wireless ad-hoc networks. Both definitions are considered inappropriate for video broadcasting in cellular networks since the transmission power is not the highest priority concern of the BS and each node might not be in the position to receive packets from different sources. To the best of our knowledge, a measurement that takes both user perspective and transmission bitrate into account for video broadcast has not been proposed. Therefore, it urges us to redefine broadcast efficiency to evaluate broadcasting strategy in cellular network. 
In this paper, we first propose a new definition of broadcast efficiency. It is the ratio between the number of served audiences and the consumed radio resources, which is the bitrate. Second, we analyze the growth rate of the number of served audiences and the required bitrate. Then, we investigate the effect of broadcasting various TV channels on broadcast efficiency. By the analysis, we found that the broadcast efficiency decreases when less popular TV channels are broadcasted. Finally, we validate our findings by numerical evaluation and a real-life simulation.

The rest of this paper is organized as follows. Section II describes the formulation of the model in detail. Numerical analysis is presented in Section III. The evaluation is demonstrated in Section IV. Finally, conclusions are made in Section V.

\section{Model Formulation}

In this section, the model formulation of the system is presented.

\section{A. TV channel properties}

In the system, we assume that each cell independently decides on choosing $q$ popular TV channels out of a set of TV channels $C$ for broadcasting. The total number of TV channels in $C$ is defined as $|C|$. The number of audiences of each channel $c \in C$ is defined as $n(c)$. The total number of audiences in the cell is $N$. The TV channels are sorted by the popularity, which is the number of audiences of each channel, in descending order. This is defined as follows,

Definition 1. $n\left(c_{1}\right) \geq n\left(c_{2}\right)$ when $c_{1}<c_{2} ; \forall c_{1}, c_{2} \in C$.

$n(c)$ is a decreasing discrete function, which implies that the most popular TV channel in the set $C$ has a larger or equal number of audiences than the second popular TV channel, and so on.

The required bitrate of each TV channel for broadcasting is defined as $r(c)$. For simplicity, and without loss of generality, we assume all of the channels have the same bitrate where,

$$
r\left(c_{1}\right)=r\left(c_{2}\right), \forall c_{1}, c_{2} \in C .
$$

\section{B. Broadcast efficiency}

The broadcast efficiency $E(q)$ is a measure for the number of users are served per unit throughput. The unit is defined as users per bit per second. The numerator of the broadcast efficiency, which is the total number of audiences for broadcasting $q$ channels, is formulated as follows,

$$
f_{n}(q)=\sum_{i=1}^{q} n(i)
$$

where $q \in C$ is the number of popular channels that are selected to be broadcasted. The denominator, which is the total required bitrates for broadcasting $q$ channel, is formulated as follows,

$$
f_{r}(q)=\sum_{i=1}^{q} r(i)
$$

Finally, the formulation of broadcast efficiency is defined as follows,

\section{Definition 2.}

$$
E(q)=\frac{f_{n}(q)}{f_{r}(q)},
$$

where $q \in C$ is the number of popular channels that are selected to be broadcasted.

A larger value of $E(q)$ indicates a higher broadcast efficiency. This indicates that either a larger number of audiences are being served or a lower bitrate is required for broadcasting. In other words, the broadcast efficiency increases when the number of watching audiences of the broadcasting channels increase or the required bitrate for broadcasting decrease.

\section{ANALYSIS}

In this section, we first analyze the growth rate of the numerator and denominator of $E(q)$ in Definition 2, which are the total number of TV channels audiences and the total required bitrate. Then, we investigate how the broadcast efficiency $E(q)$ is varied with $q$, which is the number of channels that are selected to be broadcasted.

Lemma 1. $f_{n}(q)$ is an increasing function and the upper bound of the growth rate of the function is $O(q)$.

Proof. First, we analyze the extreme case that all of the numbers of audiences of each TV channel are equal.

Case 1. If $n\left(c_{1}\right)=n\left(c_{2}\right)$, then $f_{n}(q)=q \cdot n(1)=\Theta(q)$.

In this case, the total number of audiences increases linearly when the number of TV channels being broadcasted increases. It implies that the growth rate of $f_{n}(q)$ is strictly linear. Since $n\left(c_{1}\right) \ngtr n\left(c_{2}\right)$, the upper bound of growth rate of $f_{n}(q)$ is $O(q)$.

Then, we analyze the general case that more popular channels have more audiences than less popular channels.

Case 2. If $n\left(c_{1}\right) \geq n\left(c_{2}\right)$, then $f_{n}(q) \leq q \cdot n(1)=O(q)$

Since $n(c)$ is a decreasing discrete function, the numbers of audiences of a more popular TV channels are always greater or equal to the less popular TV channels. In this case, $f_{n}(q)$ is increasing and the growth rate is slower than a linear function.

Finally, we analyze the growth rate if the values of $n(c)$ is equal to zero.

Case 3. If $\exists n\left(c_{1}\right)=0$, then $n\left(c_{2}\right)=0, \forall c_{2}>c_{1}$; $f_{n}(q) \leq f_{n}\left(c_{1}\right)=O(1)$, where $f_{n}\left(c_{1}\right)$ is a constant.

Since $n(c)$ is a decreasing discrete function, once there is a TV channel $c_{1}$ has no audience, the channels which are less popular than it, have no audience as well. Therefore, the value of $f_{n}(q)$ will not increase after $q=c_{1}$. In this case, the growth rate of $f_{n}(q)$ is upper bounded by a constant.

By summarizing the above cases, $f_{n}(q)=O(q)$ and Lemma 1 is proven. 
Lemma 2. $f_{r}(q)$ is an increasing function and the growth rate of the function is $\Theta(q)$.

Proof. From (1), the bitrates of TV channels are equal. The summation of the bitrates increases linearly with respect to $q$. It implies that the growth rate of $f_{r}(q)$ is strictly linear. It is upper bounded and lower bounded by linear growth functions. Therefore, $f_{r}(q)=\Theta(q)$.

Lemma 3. $E(q)$ is a decreasing function and the value of $E(q)$ is lower bounded by $\frac{N}{|C| r(1)}$.

Proof. $E(q)$ is composited by the numerator with $f_{n}(q)$ and the denominator with $f_{r}(q)$. From Lemma 1 and 2, we prove that $f_{n}(q)=O(q)$ and $f_{r}(q)=\Theta(q)$. Hence, $f_{n}(q)=$ $O\left(f_{r}(q)\right)$. It implies that the denominator $f_{r}(q)$ growth faster than or equal to the numerator $f_{n}(q)$. Therefore, $E(q)$ is a decreasing function.

Since $q \in C$, the maximum value of $q$ is $|C|$. The maximum value of $f_{n}(|C|)$ which is the total number of audience $N$. The maximum bitrate for broadcasting is $f_{r}(|C|)$. The fastest growth rate of the numerator is linear. When it is divided by the linear-growth denominator, the decrease rate of $E(q)$ will become slowest as a constant function and become the lower bound of $E(q)$. The lower bound value is $\frac{f_{n}(|C|)}{f_{r}(|C|)}=$ $\frac{N}{|C| r(1)}$

In summary, according to Lemma 3 , we prove that the broadcast efficiency decreases when broadcasting more and more unpopular TV channels. Furthermore, the broadcast efficiency is lower bounded by $\frac{N}{|C| r(1)}$.

\section{Evaluation}

In this section, we first numerically evaluate the analysis by applying known distributions of popularity for TV channels. Then, we conducted a real-life simulation to further evaluate the broadcast efficiency.

\section{A. Numerical evaluation}

We numerically evaluate the lemmas by applying different cases and distributions for $n(c)$, which are different distributions for the size of audience of TV channels. Reported in [13] and [14], the popularity of TV channels following Zipf-like distribution. We use Zipf distribution with parameter $s=1$ in our evaluation. In addition, we include another two distributions, Zipf-Mandelbrow and Zeta, with parameter $s=2$ and $s=3$, for comparison. Furthermore, two extreme cases are added in the evaluation. One case is all of the TV channels having the same size of audience, which is an uniform distribution. It is related to Case 1 in Lemma 1. Another case is when all of the audiences watching only the most popular TV channel, which is denoted as $n(2)=0$ in the figures and related to Case 2 in Lemma 1. For the other settings, we assume there are 2000 audiences in the cell and 10 channels are being selected to be broadcasted. The required bitrate for each TV channel is $1 \mathrm{Mb} / \mathrm{s}$.

Figure 1 shows the number of audiences for each channel. The channels in the $\mathrm{x}$-axis are sorted in a descending order

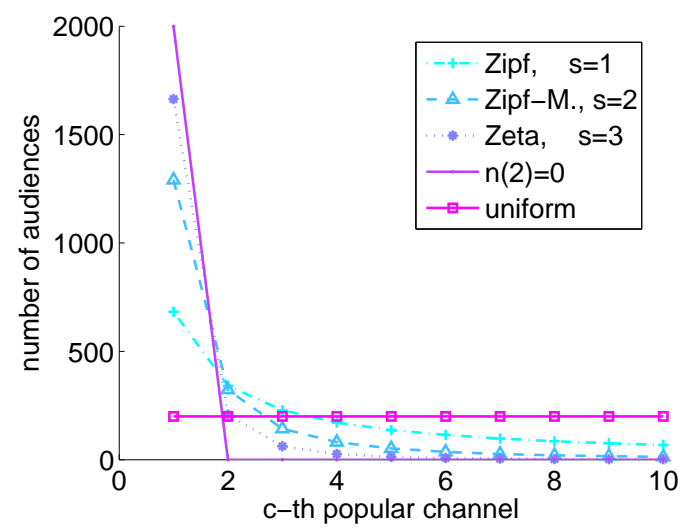

Fig. 1. Number of audience of TV channels, $\mathrm{c}$ is sorted by popularity

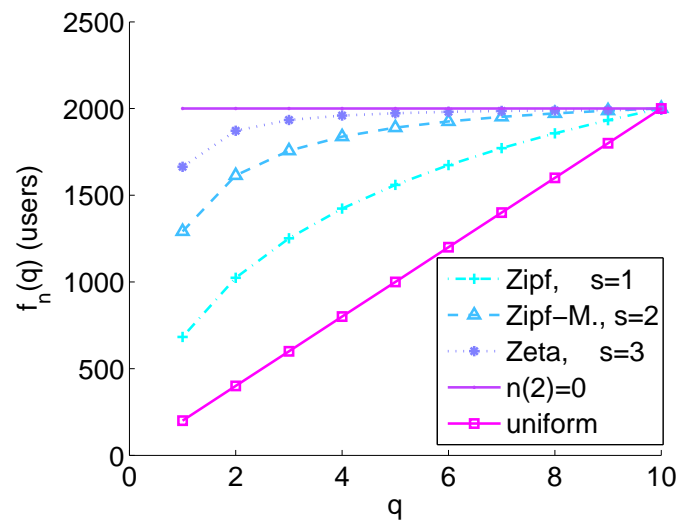

Fig. 2. $f_{n}(q)$

according to the popularity, which is the size of audience. All of the cases follows Definition 1 that the less popular channels have fewer or equal number of audiences. In Zipf distribution, the most popular TV channel has 683 audiences. The numbers of audiences drop gently to 68 audiences in the least popular channel. In Zip-Mandelbrow distribution, it starts with 1291 audiences in the most popular channel, drop quickly and negative exponentially to only 13 audiences in the least popular channel. In Zeta distribution, it starts with 1664 audiences and has a fastest drop among these three distribution to having less than 10 audiences in the last five TV channels each. In the special case that $n(2)=0$, all of the 2000 audiences watch the most popular TV channel and no audiences for the remains. For the uniform case, all of the TV channels has 200 audiences.

Figure 2 shows the function $f_{n}(q)$, which is the total number of audiences when broadcasting $q$ popular TV channels. In Zipf distribution, $f_{n}(q)$ has a low starting number of audiences and grows quickly when $q$ increases. In Zipf-Mandelbrow and Zeta distribution, both of them start with a higher number of audiences and grow slower than the Zipf distribution. In $n(2)=0$, the first TV channel has all of the 2000 audiences therefore, it remains constant and not growing in $f_{n}(q)$. In 


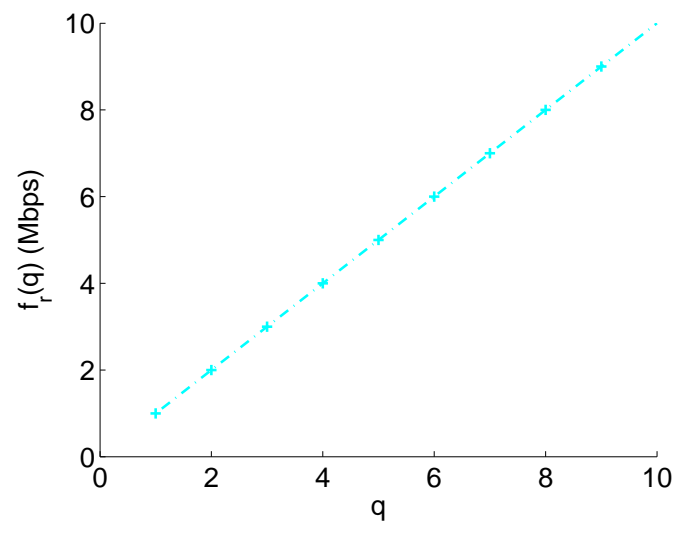

Fig. 3. $f_{r}(q)$

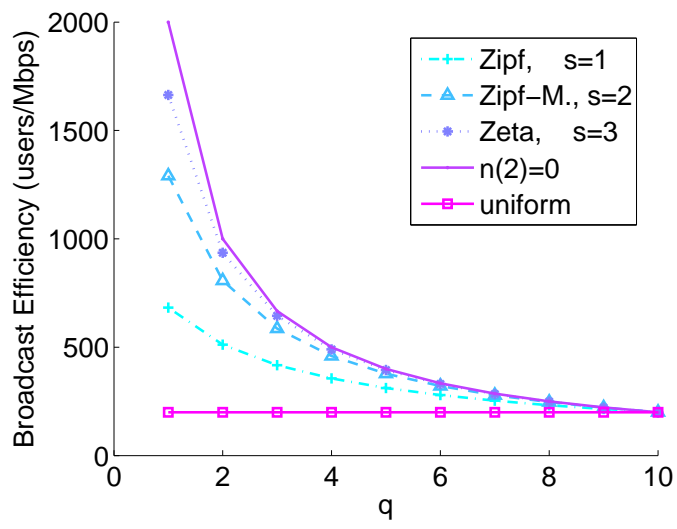

Fig. 4. Broadcast Efficiency

the uniform distribution, it grows linearly and has the fastest growth rate compare to the other cases. This validates Lemma 1 that $f_{n}(q)$ is an increasing function and the fastest growth rate of the function is linear.

Figure 3 shows the function $f_{r}(q)$, which is the total require bitrate for broadcasting $q$ TV channels. From the assumption in (1), all of the TV channels have the same bitrate. Therefore, $f_{r}(q)$ grows exactly linearly. This validates Lemma 2 that $f_{r}(q)$ is a strictly linear growth function.

Figure 4 shows the broadcast efficiency for each case. It shows that $E(q)$ is a decreasing function. The case $n(2)=$ 0 has the fastest decreasing rate since broadcasting more channels in this case does not serve any more audience. It is a waste of resources if broadcasting more than one TV channel when there is no audience in the remaining $9 \mathrm{TV}$ channels. Zipf distribution has a slower decreasing rate than the other two distributions because more audiences in the unpopular channels. It leads to a conclusion that a more even distribution of audiences on the TV channels helps to slow down the decreasing rate of broadcast efficiency when broadcasting more TV channels. In the uniform distribution, broadcast efficiency remains constant since both the numerator and denominator of $E(q)$ are linear. It is also the lower

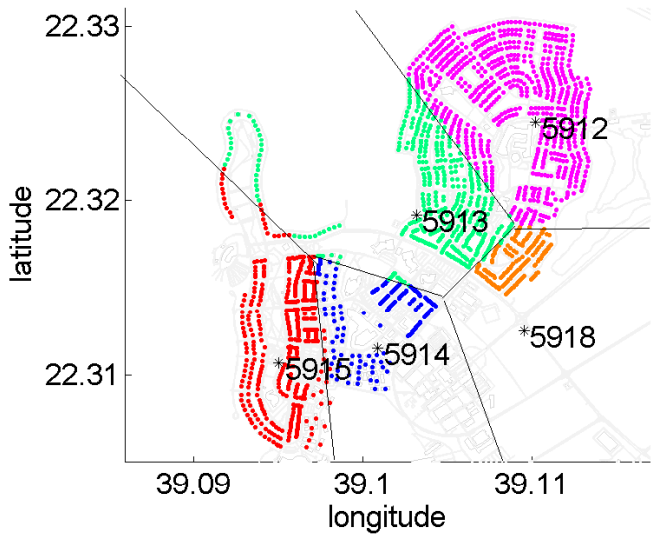

Fig. 5. BS assignment for each house and building grouped and colored by assigned BS, with the cell boundaries

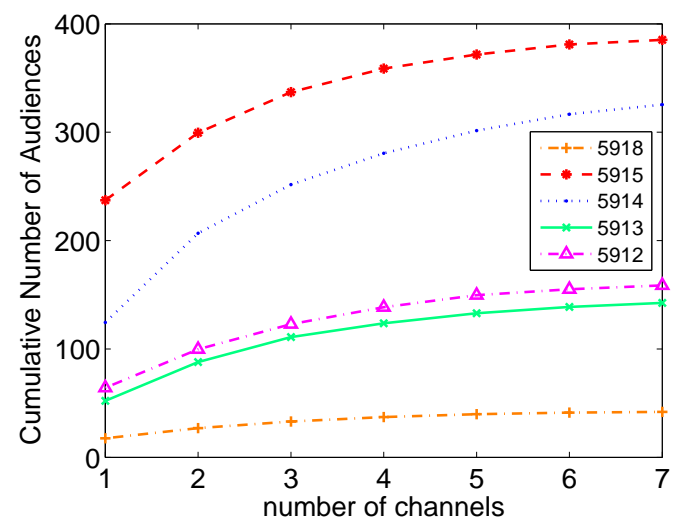

Fig. 6. Average cumulative number of audiences for TV channels in each BS coverage area

bound of the broadcast efficiency. The lower bound value is $200 \mathrm{users} / \mathrm{Mb} / \mathrm{s}$ which is equal to $\frac{N}{|C| r(1)}$ described in Lemma 3.

In summary, the numerical evaluation validates the lemmas presented in the previous section. We further show the effect of broadcasting different numbers of TV channels on broadcast efficiency in the following subsection by a real-life simulation.

\section{B. Real-life simulation}

A real-life simulation is conducted to evaluate the broadcast efficiency with various number of broadcasting channels.

1) Simulation setup: We obtained a real-life dataset from statistical data and actual geographical location for evaluating our work. The statistical data are extracted from the surveys of TV audience behavior in Saudi Arabia reported by Dubai Press Club and Pan Arab Research Center in 2007, 2011 and 2012 [15]-[17]. The daily average people using television (PUT) level presented in [17] is adopted to estimate the overall size of audience. The seven most popular TV channels are considered for simplicity. The audience sizes of the channels are approximated from [16]. We assumed that the bitrates of the TV channels are $700 \mathrm{~kb} / \mathrm{s}$. 


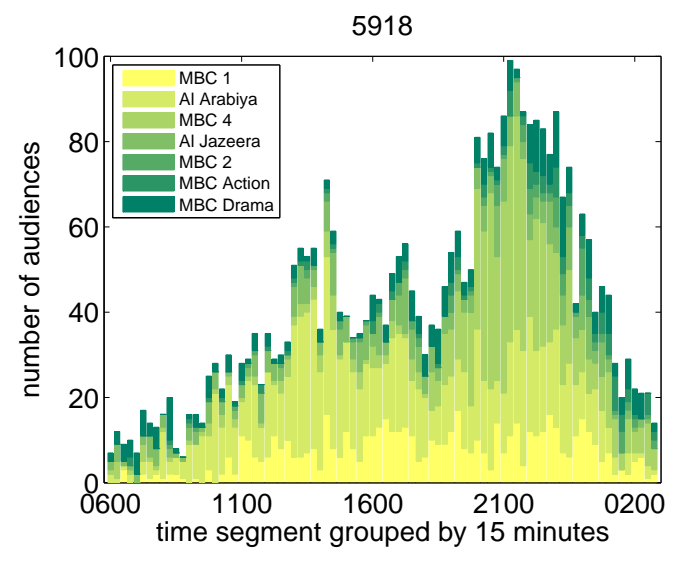

Fig. 7. Number of audiences in BS 5918

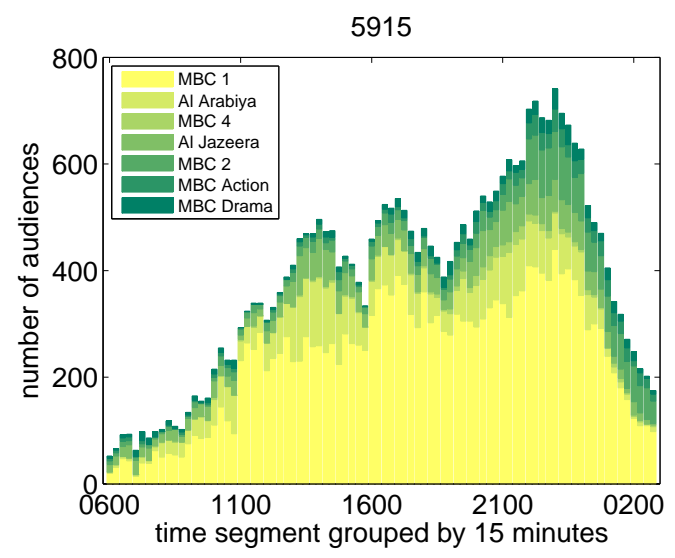

Fig. 8. Number of audiences in BS 5915

For the geographical location, we considered King Abdullah University of Science and Technology (KAUST), located in Saudi Arabia, as our study location. KAUST is a midsize living compound with about 2000 town houses and 80 apartment buildings. We assumed that there are two sets of watching equipment, such as, TV sets, tablets, or smartphones, in each town house, and twenty sets of watching equipment in each apartment building, in a total of 5490 sets. We assumed a static BS assignment for each house and building by selecting the strongest reception signal from the BS. Furthermore, there are five BSs operated by the mobile network service provider. These are shown as stars with the BS name in Figure 5. Each BS has the same transmission power and coverage area. The receivers under the cell coverage have similar channel conditions to receive fair video quality. The assignment is colored in Figure 5 according to the assigned BS. Figure 6 shows the average cumulative number of audiences for sorted TV channels, in each BS coverage area. BS 5915 has the most number of audiences in average and BS 5918 has the least number of audiences.

2) Simulation result: Two BSs which have the most and the least number of audiences, BS 5915 and BS 5918, respectively, are selected to be discussed in this paper.

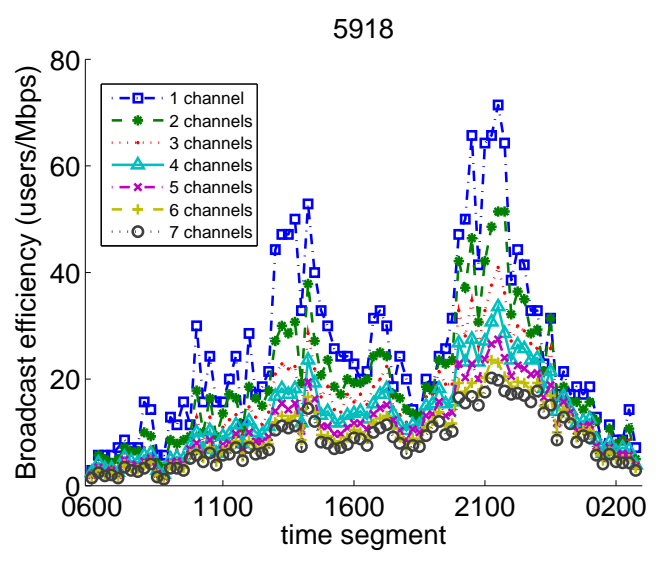

Fig. 9. Broadcast efficiency in BS 5918

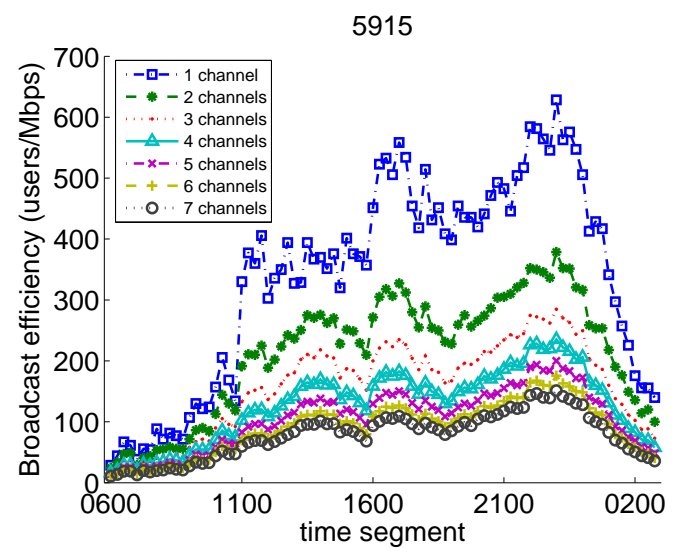

Fig. 10. Broadcast efficiency in BS 5915

In Figure 7 and 8, the number of audiences of each channel in a day from 6AM to $2 \mathrm{AM}$ are shown. The data are stacked in the bar charts and grouped by 15 minutes. These two BSs shows a big difference in the audience distribution. BS 5918 has more audiences for Al Arabiya across the day and more audiences for MBC 4 in the evening. BS 5915 is mainly dominated by the audiences for MBC 1 across the day.

Figure 9 and 10 show the broadcasting efficiency of these two BSs. The figures have seven cases which show the broadcasting efficiency for broadcasting different numbers of TV channels. This validates our finding that broadcasting more channels decreases the broadcast efficiency. Furthermore, by comparing these two figures, it clearly shows that the difference of audience distributions affect the broadcast efficiency. First, the average value of broadcast efficiency of BS 5918 is lower than BS 5915 due to the lower number of average total audiences. It shows that the total number of audiences is directly proportional to the broadcast efficiency. Second, the differences of broadcast efficiency between broadcasting more channels in BS 5915 is larger than the differences in BS 5918. For example, at 21:30, the difference of broadcasting 1 channel and 2 channels in BS 5918 drops $18.7 \%$, but in BS 5915, it drops $28.2 \%$. This is because BS 5915 has a larger portion 


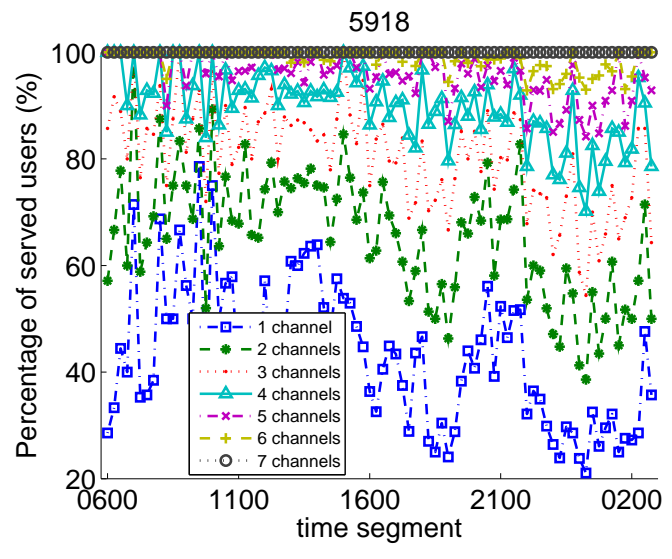

Fig. 11. Percentage of served audiences in BS 5918

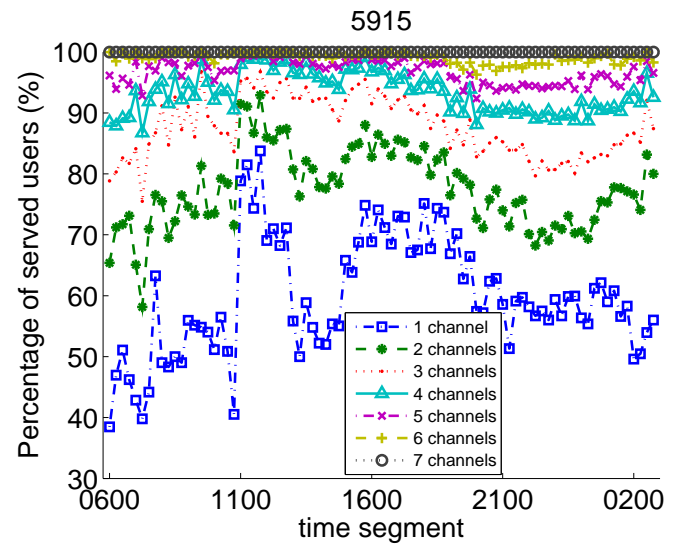

Fig. 12. Percentage of served audiences in BS 5915

of audiences watching the most popular TV channel than in BS 5918, where the audiences are more evenly distributed. By this observation, we summarize that broadcasting more unpopular channels in audience uneven distributed cells drops the broadcast efficiency faster.

However, there is a trade-off for high broadcast efficiency. Figure 11 and 12 show the percentage of served audiences for different numbers of broadcasting channels. The fewer TV channels are broadcasted, the higher broadcast efficiency, but a lower number of served audiences. In BS 5918, since the distribution of audiences between different TV channels is more even, broadcasting only one popular channel can serve only $42.9 \%$ of audience in average. However, in average, $59.8 \%$ of total audiences are served in BS 5915 when broadcasting only the most popular channel. In order to guarantee that more than $90 \%$ of audiences are served at any time, BS 5918 needs to broadcast at least $6 \mathrm{TV}$ channels to fulfill the requirement. In BS 5915, it only requires $5 \mathrm{TV}$ channels to serve more than $90 \%$ of the audiences. Broadcasting 3 TV channels can mostly serve $80 \%$ of the requirement. In summary, more audiences can be served when broadcasting fewer TV channels if the audience distribution is not evenly distributed, which indicates that the difference of audiences between popular and unpopular TV channels should be as large as possible.

\section{CONCLUSION}

In this paper, we defined a new metric known as the user broadcast efficiency. It measures the size of audience served when a cell is broadcasting TV channels in terms of consumed bitrates. Numerical analysis and evaluation proved that broadcast efficiency decreases when broadcasting less popular TV channels. By conducting a real-life simulation, we demonstrated that high broadcast efficiency comes with a low percentage of served audiences as a trade-off. In the future, we will investigate the effects of broadcast efficiency by varying TV channel bitrates. Furthermore, we will balance between broadcast efficiency and the number of served audiences as an essential target for future research.

\section{REFERENCES}

[1] D. Lecompte and F. Gabin, "Evolved multimedia broadcast/multicast service (eMBMS) in LTE-advanced: overview and Rel-11 enhancements," IEEE Commun. Mag., vol. 50, no. 11, pp. 68-74, 2012.

[2] Mobily, "mView entertainment application," 2014. [Online]. Available: http://mview.mobily.com.sa/

[3] M. Learmonth, "Verizon: We're Planning To Use Our 4G LTE Network For Wireless TV," 2014. [Online]. Available: http://www.ibtimes.com/ verizon-were-planning-use-our-4g-lte-network-wireless-tv- 1655296

[4] Nokia, "Nokia Networks first to trial LTE for national TV broadcasting," 2014. [Online]. Available: http://networks.nokia.com/news-events/press-room/press-releases/ nokia-networks-first-to-trial-lte-for-national-tv-broadcasting

[5] C.-X. Wang, F. Haider, X. Gao, X.-H. You, Y. Yang, D. Yuan, H. Aggoune, H. Haas, S. Fletcher, and E. Hepsaydir, "Cellular architecture and key technologies for $5 \mathrm{~g}$ wireless communication networks," Communications Magazine, IEEE, vol. 52, no. 2, pp. 122-130, February 2014.

[6] L. Shi, K. W. Sung, and J. Zander, "Spectrum requirement for cellular tv distribution in uhf band from urban to rural environment," in $D y$ namic Spectrum Access Networks (DYSPAN), 2014 IEEE International Symposium on, April 2014, pp. 362-365.

[7] C. P. Lau, K. Jamshaid, and B. Shihada, "Efficient power allocation for video over superposition coding," in IEEE Int. Conf. on Advanced Inform. Networking and Appl. (AINA), Mar. 2013, pp. 808-813.

[8] C. P. Lau, B. Shihada, and P.-h. Ho, "Empirical evaluation of superposition coded multicasting for scalable video," in IEEE Int. Conf. on Advanced Inform. Networking and Appl. Workshop (WAINA), 2013, pp. 566-571.

[9] C. P. Lau, X. Zhang, and B. Shihada, "Video quality prediction over wireless 4G," in Pacific-Asia Conf. on Knowledge Discovery and Data Mining (PAKDD), vol. 7819, 2013, pp. 414-425.

[10] F. Ye, R. Yim, S. Roy, and J. Zhang, "Efficiency and reliability of one-hop broadcasting in vehicular ad hoc networks," Selected Areas in Communications, IEEE Journal on, vol. 29, no. 1, pp. 151-160, 2011.

[11] F. Ye, R. Yim, J. Zhang, and S. Roy, "Congestion control to achieve optimal broadcast efficiency in vanets," in Communications (ICC), 2010 IEEE International Conference on, May 2010, pp. 1-5.

[12] I. Kang and R. Poovendran, "A novel power-efficient broadcast routing algorithm exploiting broadcast efficiency," in Vehicular Technology Conference, 2003. VTC 2003-Fall. 2003 IEEE 58th, vol. 5. IEEE, 2003, pp. 2926-2930.

[13] T. Qiu, Z. Ge, S. Lee, J. Wang, Q. Zhao, and J. Xu, "Modeling channel popularity dynamics in a large iptv system," SIGMETRICS Perform. Eval. Rev., vol. 37, no. 1, pp. 275-286, Jun. 2009.

[14] M. Cha, P. Rodriguez, J. Crowcroft, S. Moon, and X. Amatriain, "Watching television over an ip network," in Proceedings of the 8th ACM SIGCOMM Conference on Internet Measurement, ser. IMC '08. New York, NY, USA: ACM, 2008, pp. 71-84.

[15] Dubai Press Club, “Arab Media Outlook 2011-2015,” Tech. Rep., 2012.

[16] IPSOS and Q.media, "Al Jazeera Arabic (AJA): facts and figures, Saudi Arabia 2011,” 2011.

[17] Pan Arab Research Center, "TV audience ratings: September Y2007 continuous TV ratings survey in Kingdom of Saudi Arabia," 2007. 